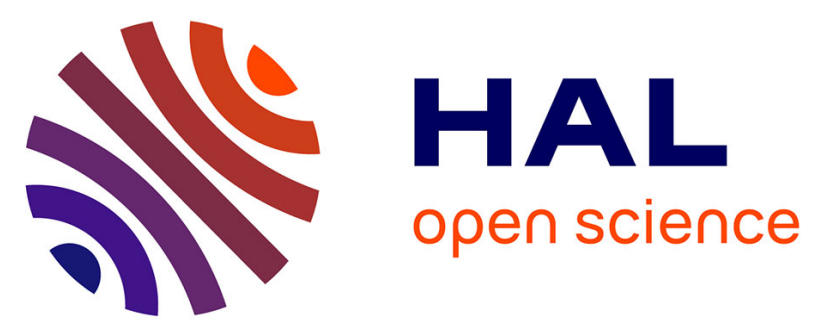

\title{
Morphology Transition of $\mathrm{ZnO}$ from Thin Film to Nanowires on Silicon and its Correlated Enhanced Zinc Polarity Uniformity and Piezoelectric Responses
}

Quang Chieu Bui, Gustavo Ardila, Eirini Sarigiannidou, Hervé Roussel, Carmen Jiménez, Odette Chaix-Pluchery, Youssouf Guerfi, Franck Bassani, Fabrice Donatini, Xavier Mescot, et al.

\section{To cite this version:}

Quang Chieu Bui, Gustavo Ardila, Eirini Sarigiannidou, Hervé Roussel, Carmen Jiménez, et al.. Morphology Transition of ZnO from Thin Film to Nanowires on Silicon and its Correlated Enhanced Zinc Polarity Uniformity and Piezoelectric Responses. ACS Applied Materials \& Interfaces, 2020, 12 (26), pp.29583-29593. 10.1021/acsami.0c04112 . hal-02917568

\section{HAL Id: hal-02917568 \\ https://hal.univ-grenoble-alpes.fr/hal-02917568}

Submitted on 26 Nov 2020

HAL is a multi-disciplinary open access archive for the deposit and dissemination of scientific research documents, whether they are published or not. The documents may come from teaching and research institutions in France or abroad, or from public or private research centers.
L'archive ouverte pluridisciplinaire HAL, est destinée au dépôt et à la diffusion de documents scientifiques de niveau recherche, publiés ou non, émanant des établissements d'enseignement et de recherche français ou étrangers, des laboratoires publics ou privés. 


\title{
Morphology Transition of $\mathrm{ZnO}$ from Thin Film to Nanowires on \\ Silicon and its Correlated Enhanced Zinc Polarity Uniformity and \\ Piezoelectric Responses
}

Quang Chieu Bui ${ }^{1,2,3}$ Gustavo Ardila, ${ }^{2}$ Eirini Sarigiannidou, ${ }^{1}$ Hervé Roussel, ${ }^{1}$ Carmen Jiménez, ${ }^{1}$ Odette Chaix-Pluchery, ${ }^{1}$ Youssouf Guerfi, ${ }^{3}$ Franck Bassani, ${ }^{3}$ Fabrice Donatini, ${ }^{4}$ Xavier Mescot, ${ }^{2}$ Bassem Salem, ${ }^{3}$ and Vincent Consonni. ${ }^{1 *}$

${ }^{1}$ Université Grenoble Alpes, CNRS, Grenoble INP, LMGP, F-38000 Grenoble, France.

${ }^{2}$ Université Grenoble Alpes, CNRS, Grenoble INP, IMEP-LAHC, F-38000 Grenoble, France.

${ }^{3}$ Université Grenoble Alpes, CNRS, LTM, F-38054 Grenoble Cedex, France.

${ }^{4}$ Université Grenoble Alpes, CNRS, Grenoble INP, Institut NEEL, F-38000 Grenoble, France.

*Corresponding author: vincent.consonni@grenoble-inp.fr

\begin{abstract}
$\mathrm{ZnO}$ thin films and nanostructures have received over the last decade an increasing interest in the field of piezoelectricity, but their formation mechanisms on silicon when using pulsed-liquid injection metalorganic chemical vapor deposition (PLI-MOCVD) are still open to a large extent. Also, the effects of their morphology, dimensions, polarity and electrical properties on their piezoelectric properties have not been completely decoupled yet. By only tuning the growth temperature from 400 to $750{ }^{\circ} \mathrm{C}$ while fixing the other growth conditions, the morphology transition of $\mathrm{ZnO}$ deposits on silicon from stacked thin films to nanowires through columnar thin films is shown. A detailed analysis of their formation mechanisms is further provided. The present transition is associated with a strong enhancement of their crystallinity and growth texture along the $c$-axis together with a massive relaxation of the strain in nanowires. It is also related to a prevailed zinc polarity, for which its uniformity is strongly improved in nanowires. The nucleation of basal-plane stacking faults of $\mathrm{I}_{1}$-type in nanowires is also revealed and related to an emission line around $3.326 \mathrm{eV}$ in cathodoluminescence, further exhibiting a fairly low
\end{abstract}


phonon coupling. Interestingly, the transition is additionally associated with a significant improvement of the piezoelectric amplitude, as determined by piezo-response force microscopy measurements. The Zn-polar domains further exhibit a larger piezoelectric amplitude than the O-polar domains, showing the importance of controlling the polarity in these deposits as a prerequisite to enhance the performances of the piezoelectric devices. The present findings demonstrate the high potential in using the PLIMOCVD system to form $\mathrm{ZnO}$ with different morphologies and a polarity uniformity on silicon. They further reveal unambiguously the superiority of nanowires over thin films for piezoelectric devices.

KEYWORDS: Zinc Oxide, Metal-Organic Chemical Vapor Deposition, Nanostructures, Morphology Transition, Piezoelectricity, Polarity. 


\section{INTRODUCTION}

$\mathrm{ZnO}$ is an abundant, biocompatible semiconductor compound offering a large number of assets that are of high interest for a broad range of applications. ${ }^{1-2}$ It has a direct wide band gap energy of $3.37 \mathrm{eV}$ at $300 \mathrm{~K}$ and a large exciton binding energy of $60 \mathrm{meV}$, which are ideal properties for UV light emitting diodes and lasers. ${ }^{3}$ Its high electron mobility of about $200 \mathrm{~cm}^{2} / \mathrm{V} . \mathrm{s}$ is also beneficial for its use as an electron transporting material in solar cells and self-powered UV photodetectors. ${ }^{4-5}$ Its noncentrosymmetric wurtzite structure further confers piezoelectric properties along the $c$-axis, giving rise to its possible integration into piezoelectric devices including nano-generators and pressure/strain sensors. ${ }^{6-9}$ In that field, the magnitude of the piezo-potential generated under mechanical constraint strongly depends on the morphology of $\mathrm{ZnO}$ deposits (i.e. dimensions, texture along the $c$-axis, polarity) as well as their electrical properties (i.e. electron density). Its high thermal stability represents another advantage, giving the capability of using $\mathrm{ZnO}$ deposits in harsh environment.

$\mathrm{ZnO}$ can be synthesized in the form of thin films and nanostructures by various physical and chemical deposition techniques including thermal evaporation, ${ }^{10}$ radio-frequency magnetron sputtering, ${ }^{11}$ pulsed laser deposition, ${ }^{12}$ molecular beam epitaxy, ${ }^{13}$ sol-gel process, ${ }^{14}$ chemical bath deposition, ${ }^{15}$ electrodeposition, ${ }^{16}$ atomic layer deposition, ${ }^{17}$ and metal-organic chemical vapor deposition (MOCVD). ${ }^{18-20}$ This broad variety of deposition techniques to form $\mathrm{ZnO}$ is unique in the field of semiconductor compounds. Among them, the MOCVD process is a chemical route to produce $\mathrm{ZnO}$ over large surface area in a fast manner and does not require high vacuum while still achieving a high structural and optical quality. The MOCVD process, however, involves a large number of parameters to be optimized such as growth temperature, ${ }^{21}$ total pressure, flow rate, ${ }^{22}$ and nature of precursors, ${ }^{23}$ which basically affect, to a large extent, the formation mechanisms of $\mathrm{ZnO}$ thin films and nanostructures. The substrate nature also plays a significant role. ${ }^{24}$ In particular, the growth temperature is an essential parameter that can strongly influence the MOCVD process in many ways. The growth temperature directly affects the growth rate of $\mathrm{ZnO}$ deposits. Basically, it favors the decomposition of chemical precursors and enhances the diffusion, adsorption, and desorption of ad-atoms on the substrate while boosting the chemical reaction. This in turn influences the formation mechanisms as well as the 
properties of $\mathrm{ZnO}$ deposits. ${ }^{21,23}$ By varying the growth temperature from 250 to $720^{\circ} \mathrm{C}$, different growth regimes in MOCVD involving the enhancement and degradation of the structural, optical, and electrical properties of $\mathrm{ZnO}$ films were reported in Ref. ${ }^{25}$. The influences of the growth temperature on the premature coalescence of nuclei and growth texture were also demonstrated in Ref. ${ }^{26}$. The improvement of the crystallinity of $\mathrm{ZnO}$ deposits on quartz was also found by raising the growth temperature. ${ }^{27} \mathrm{~A}$ double step growth involving the formation of an initial layer at $350{ }^{\circ} \mathrm{C}$ to decrease the magnitude of the premature coalescence process, and then a subsequent growth at $400{ }^{\circ} \mathrm{C}$ was further developed. ${ }^{28} \mathrm{In}$ such a way, $\mathrm{ZnO}$ films were shown to exhibit an improved growth texture as compared to the single step growth either at 350 or $400{ }^{\circ} \mathrm{C}$ Additionally, the morphology transition of $\mathrm{ZnO}$ from thin films to nanowires on sapphire was revealed when the growth temperature raises from 200 to $380{ }^{\circ} \mathrm{C}$. This was attributed to the increase in the ad-atom diffusion enhancing in turn the anisotropic growth. Surprisingly, in contrast to the thermal evaporation and chemical bath deposition techniques, the MOCVD process has not extensively been used to deposit $\mathrm{ZnO}$ in different forms for piezoelectric devices, while its integration over a silicon wafer and its effect on the formation mechanisms has not been completely elucidated yet. Moreover, most of the works have reported the use of a MOCVD reactor with a bubbler system for the growth of $\mathrm{ZnO}$ thin films and nanowires. In contrast, the pulsed-liquid injection MOCVD (PLI-MOCVD) reactor appears to be of high interest by mixing the zinc chemical precursor in a solvent and by typically working at a lower growth temperature.

In this work, the morphology transition of $\mathrm{ZnO}$ from thin film to nanowires grown by PLI-MOCVD is achieved on $\mathrm{Si}$ substrates by tuning the growth temperature in the range of $400-750{ }^{\circ} \mathrm{C}$. The structural, optical, electrical, and piezoelectric properties of $\mathrm{ZnO}$ deposits are investigated in details by using field-emission gun scanning electron microscopy (FESEM), transmission electron microscopy (TEM), x-ray diffraction (XRD), Raman spectroscopy, cathodoluminescence (CL) and piezo-response force microscopy (PFM) measurements. The formation mechanisms and structural properties are discussed in details while showing that the morphology transition of $\mathrm{ZnO}$ from thin films to nanowires is highly beneficial to enhance their optical and piezoelectric properties.

\section{MATERIALS AND METHODS}


2.1. ZnO thin film and nanowire growth. $\mathrm{ZnO}$ thin films and nanowires were grown on $\mathrm{Si}$ (100) wafers by PLI-MOCVD in an Annealsys MC-200 MOCVD system. Diethylzinc $\left(\mathrm{Zn}\left(\mathrm{C}_{2} \mathrm{H}_{5}\right)_{2}\right.$, DEZn, Sigma-Aldrich) with a concentration of $1 \mathrm{M}$ was diluted in cyclohexane as the zinc chemical precursor solution while $\mathrm{O}_{2}$ molecules were used as the oxygen agent. The flow rates of DEZn solution and $\mathrm{O}_{2}$ molecules were set to $0.5 \mathrm{~g} / \mathrm{min}$ and $500 \mathrm{sccm}$, respectively. This corresponds to an $\mathrm{O} / \mathrm{Zn}$ ratio of about 100. Argon with a flow rate of $500 \mathrm{sccm}$ was used as gas carrier for the DEZn chemical precursor. The chamber pressure was maintained at 3 mbar during the MOCVD process. The temperature of the substrate was varied in the range of 400 to $750{ }^{\circ} \mathrm{C}$.

2.2. Structural property measurements. Cross-sectional- and top-view FESEM images of $\mathrm{ZnO}$ thin films and nanostructures were recorded with a Quanta 250 FEI FEG-SEM. XRD measurements were collected in the range of 20 to $140^{\circ}$ in $2 \theta$-scale with a Bruker D8 Advance diffractometer using $\mathrm{CuK \alpha _{1 }}$ radiation according to the Bragg-Brentano configuration. The texture coefficients $C_{\mathrm{hkl}}$ of $\mathrm{ZnO}$ thin films and nanostructures for a given (hkl) plane were determined in percent unit by the following equation: ${ }^{29}$

$$
C_{h k l}(\%)=\frac{\frac{I_{h k l} l}{I_{0, h k l}} \times 100}{\sum_{i=0}^{N} \frac{I_{h_{i} k_{i} l_{i}}}{I_{0, h_{i} k_{i} l_{i}}}}
$$

where $\mathrm{I}_{\mathrm{hkl}}$ is the hkl peak intensity, $\mathrm{I}_{0, \mathrm{hkl}}$ is the reference hkl peak intensity from the 00-036-1451 file of the International Center for Diffraction Data (ICDD), and $\mathrm{N}$ is the number of peaks considered $(\mathrm{N}=6$ here).

TEM and high-resolution TEM (HRTEM) images were collected with a JEOL $2010 \mathrm{LaB}_{6}$ microscope operating at $200 \mathrm{kV}$ with a $0.19 \mathrm{~nm}$ point-to-point resolution. TEM lamellas were prepared in cross-section by tripod polishing followed by argon ion milling according to standard techniques. Raman scattering measurements were recorded at room temperature using a Jobin Yvon/Horiba Labram spectrometer equipped with a liquid nitrogen cooled charge-coupled device (CCD) detector. The 488 $\mathrm{nm}$ excitation line of an $\mathrm{Ar}^{+}$laser with a power around $1 \mathrm{~mW}$ at the sample surface was used. The light was focused to a spot size smaller than $1 \mu \mathrm{m}^{2}$ using a 50x long working distance. Raman spectra were measured in cross-polarization in order to reduce the signal coming from the silicon substrate. They 
were calibrated at room temperature using a silicon reference sample with a theoretical position of 520.7 $\mathrm{cm}^{-1}$

2.3. Optical property measurements. $5 \mathrm{~K} \mathrm{CL}$ measurements were achieved with an FEI Inspect F50 FESEM instrument equipped with a liquid helium-cooled stage. The CL signal was collected through a parabolic mirror and analyzed with a $550 \mathrm{~nm}$ focal length monochromator equipped with a diffraction grating of 600 grooves $/ \mathrm{mm}$. The CL spectra were recorded with a thermoelectrically cooled silicon CCD detector.

\subsection{Piezoelectric property and polarity measurements. PFM measurements were} performed on thin films and nanowires using a Bruker Dimension Icon atomic force microscope (AFM). The heavily p-type doped $\mathrm{Si}(100)$ substrates were used as bottom electrical contact. The samples were directly put on the chuck of the AFM system. During the scanning, an AFM metallic tip was in contact with the sample and an $\mathrm{AC}$ voltage was applied between the tip and the chuck. Owing to the piezoelectric properties of $\mathrm{ZnO}$, the $\mathrm{AC}$ voltage induced a deformation of the sample with certain amplitude and phase, which were detected by the AFM tip. The phase in particular can be in-phase or $180^{\circ}$ out-ofphase with the applied voltage, which corresponds to the polarity of the sample. The applied AC voltage during the scanning was set to $5 \mathrm{~V}$ and the frequency was chosen at $15 \mathrm{kHz}$ to avoid the contact resonance frequency (above $200 \mathrm{kHz}$ ) and high background signal contribution, based on the piezoresponse versus frequency graphs in Figures S1 and S2. The background signal contribution to PFM measurement has been reported in Ref. ${ }^{30}$. The tips used for PFM characterizations were PtSi-NCH tips from Nanosensors with spring constant in the range of $43-50 \mathrm{~N} / \mathrm{m}$. By using a tip with high stiffness, the interference of the electrostatic force with the piezoelectric signals can greatly be reduced ${ }^{31}$ (see supplementary information and Figure S3). However, high stiffness tip also has drawbacks, such as low deflection sensitivity (in the range $59-64 \mathrm{~nm} / \mathrm{V}$ ) and the possibility to scratch or bend the thin film or nanowires, resulting in contact failure between the tip and the sample. To avoid this last problem, instead of using the classical contact mode to scan the sample, we used the DataCube PFM mode. In DataCube PFM, the tip sequentially approached, touched the sample, and withdrawn at specific chosen positions of the sample, eventually allowing mapping. For each position, the tip stayed in contact for 100 
milliseconds to collect piezoelectric signals. The amplitude and phase of each position presented here are the mean values collected in this time frame. The nanowire sample was prepared following a specific process before PFM measurements to avoid artifacts, in particular when using the AFM contact mode. Two different types of polymethyl methacrylate (A2 and A6 from MicroChem 495PMMA) were spin coated over the nanowires to ensure their complete immersion into the polymer. ${ }^{8}$ PMMA A2 having a low viscosity easily penetrate between the nanowires, then A6 with a higher viscosity is used for planarization. The polymer was then etched by reactive ion etching (RIE) under $\mathrm{Ar} / \mathrm{O}_{2}$ for 20 minutes at $60 \mathrm{~W}$ until the nanowire tips were visible, as shown in Figure S4.

2.5. Electrical property measurements. The resistivity measurements of $\mathrm{ZnO}$ samples (i.e. stacked and columnar thin films) were carried out using transmission line measurement (TLM) method. The contact electrodes were fabricated using photolithography and lift-off processes. After development step, $50 \mathrm{~nm}$-thick nickel (Ni) and $120 \mathrm{~nm}$-thick gold ( $\mathrm{Au}$ ) layers were deposited on the samples using an e-beam evaporator, followed by a lift-off step to remove photoresist. The series of rectangle electrodes with dimensions around $196 \mu \mathrm{m}$ in width (W) and $92 \mu \mathrm{m}$ in length (L), along with different distances (d) between them, were formed on the $\mathrm{ZnO}$ surfaces, as shown in Figure S5. Then, the electrical measurements were proceeded using a Karl Suss PM8 system. During the process, a current was applied and a voltage was measured between each pair of electrodes. The resistance $\left(\mathrm{R}_{\text {Total }}\right)$ obtained from the I-V curve of each electrode pair is composed of the metallic electrode resistance $\left(R_{m}\right)$, the contact resistance $\left(\mathrm{R}_{\mathrm{c}}\right)$ at electrode/thin film interface and the thin film resistance between the electrode pair, which can be interpreted as follows:

$$
R_{\text {Total }}=2 \cdot R_{m}+2 \cdot R_{c}+\frac{R_{s}}{W} \cdot d
$$

where $R_{s}$ is the sheet resistance of the thin film. The variation of total resistance $\left(R_{\text {Total }}\right)$ depending on the distance (d) between the electrode pair was recorded. The slope of linear fit to these data points was used to extract the sheet resistances as well as the resistivity of thin films.

\section{RESULTS}


3.1. Morphology transitions of $\mathrm{ZnO}$ deposits. The morphology of $\mathrm{ZnO}$ deposits grown by PLI-MOCVD and its evolution as a function of growth temperature is presented in Figure $\mathbf{1}$ by topview and cross-sectional-view FESEM images.
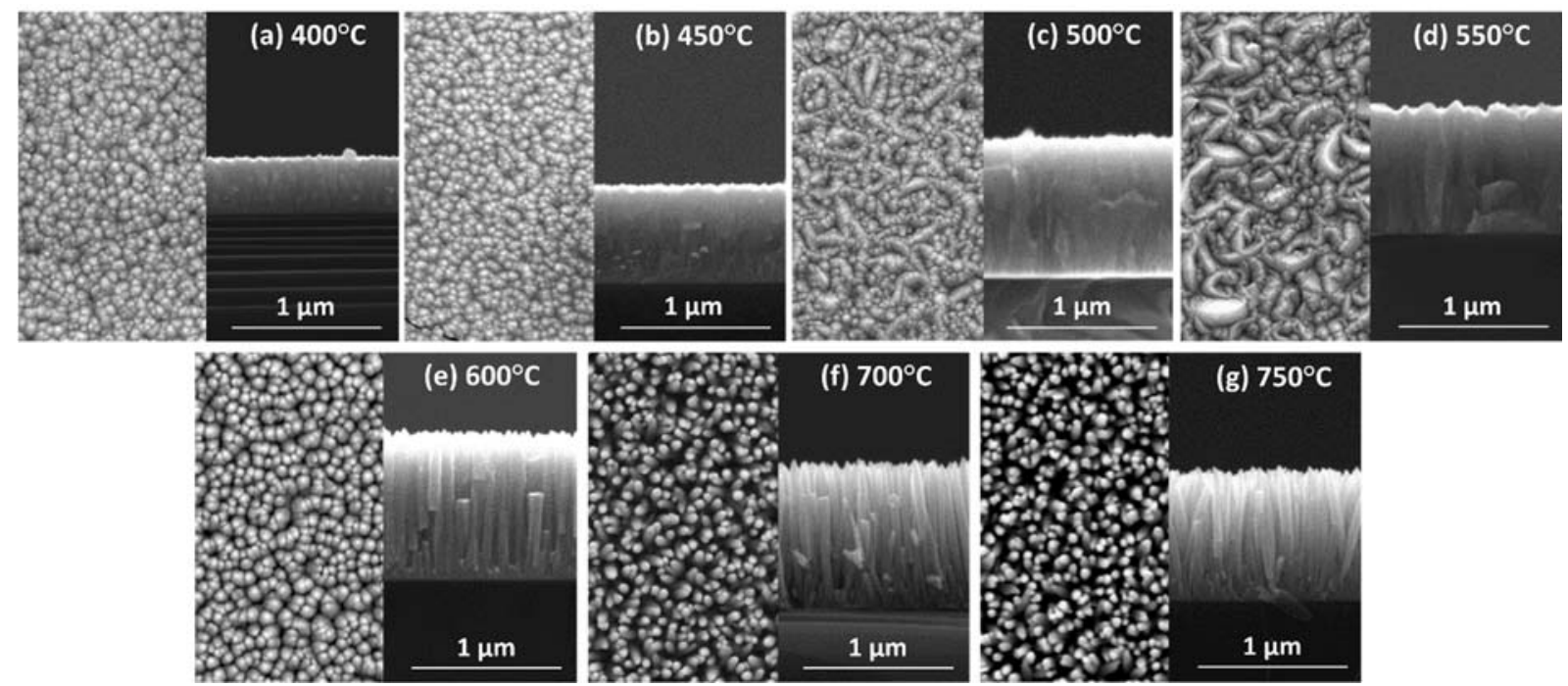

Figure 1. Top- (left) and cross-sectional-view (right) FESEM images of ZnO deposits grown by PLIMOCVD for a given growth temperature of (a) 400, (b) 450, (c) 500, (d) 550, (e) 600, (f) 700, and (g) $750{ }^{\circ} \mathrm{C}$.

In the range of 400 to $550{ }^{\circ} \mathrm{C}$, the $\mathrm{ZnO}$ deposit typically consists of a standard thin film composed of stacked grains, as shown in Figures 1a-d. Although the structure of these $\mathrm{ZnO}$ stacked thin films is quite similar in that growth temperature range, the mean grain size increases from 51 to $60 \mathrm{~nm}$ as growth temperature is raised. This is further marked by a pronounced faceting process at their top surface, resulting in the formation of highly elongated grains. At $600{ }^{\circ} \mathrm{C}$, the morphology of $\mathrm{ZnO}$ deposit starts changing from a stacked thin film to a columnar thin film, instead of continuing the increase in grain size. The columnar thin film is composed of vertically aligned coalesced nanocolumns, as revealed in Figure 1e. At higher growth temperatures of 700 and $750{ }^{\circ} \mathrm{C}$, the morphology of $\mathrm{ZnO}$ deposits is characterized by dense arrays of isolated nanowires, as shown in Figures 1f-g, respectively. Their number density is slightly decreased from $1.73 \times 10^{10}$ to $1.48 \times 10^{10}$ nanowires $/ \mathrm{cm}^{2}$ as growth temperature is raised. 


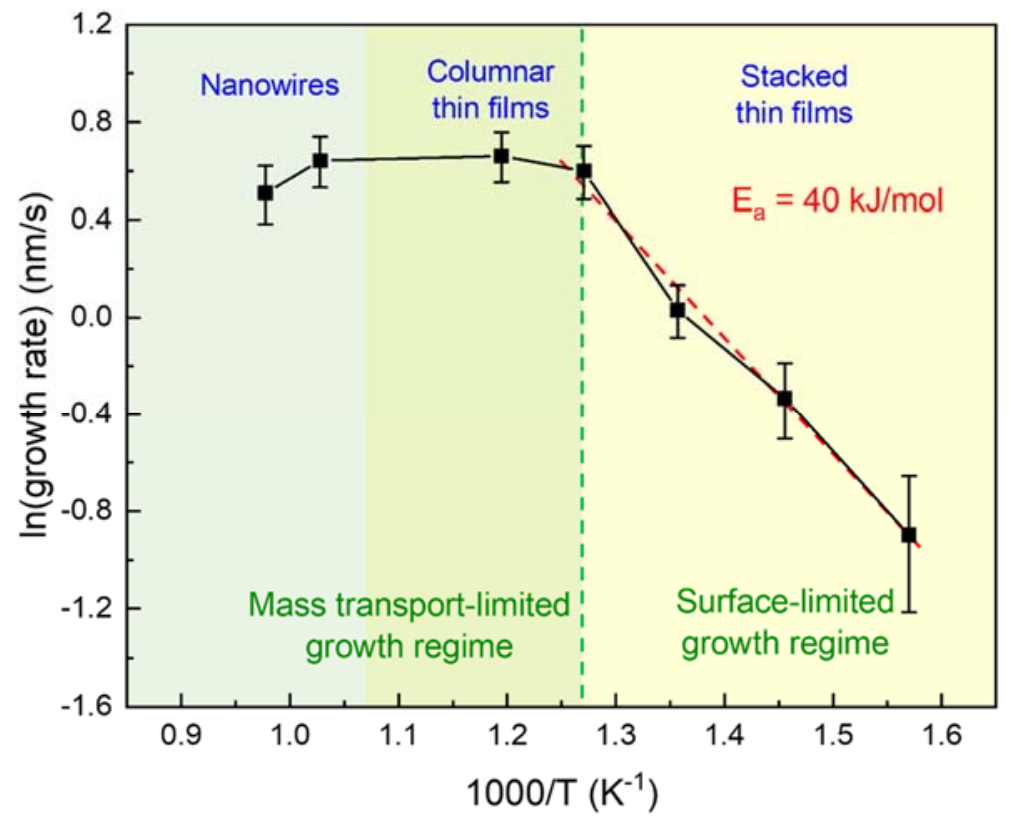

Figure 2. Growth rate of $\mathrm{ZnO}$ deposits grown by PLI-MOCVD vs reciprocal growth temperature.

The influence of growth temperature on the growth rate of $\mathrm{ZnO}$ deposits grown by PLI-MOCVD is presented in Figure 2. The regions delineating the typical surface- and mass transport-limited growth regimes in the MOCVD system are also revealed. The mean growth rate was calculated by the ratio of film thickness over growth time, or by the ratio of nanowire length over growth time. From 400 to 550 ${ }^{\circ} \mathrm{C}$, the growth rate of stacked thin films strongly increases from 0.4 to $1.9 \mathrm{~nm} / \mathrm{s}$, indicating the occurrence of a surface reaction-limited growth regime. By fitting the experimental data with an Arrhenius plot, an activation energy around $40 \mathrm{~kJ} / \mathrm{mol}$ was deduced from the slope of the linear part, which is similar to the value of $38.4 \mathrm{~kJ} / \mathrm{mol}$ reported in Ref. ${ }^{32}$. In the range of 550 to $700{ }^{\circ} \mathrm{C}$, the growth rate of columnar thin films and nanowires stops increasing and basically saturates to the value of 1.9 $\mathrm{nm} / \mathrm{s}$, indicating the occurrence of a mass transport-limited growth regime. At the high growth temperature of $750{ }^{\circ} \mathrm{C}$, the growth rate of nanowires starts decreasing to the value of $1.7 \mathrm{~nm} / \mathrm{s}$. The correlated decrease in the mean volume of $\mathrm{ZnO}$ deposits indicates the pronounced desorption of $\mathrm{Zn}$ and $\mathrm{O}$ ad-atoms at that high growth temperature. 


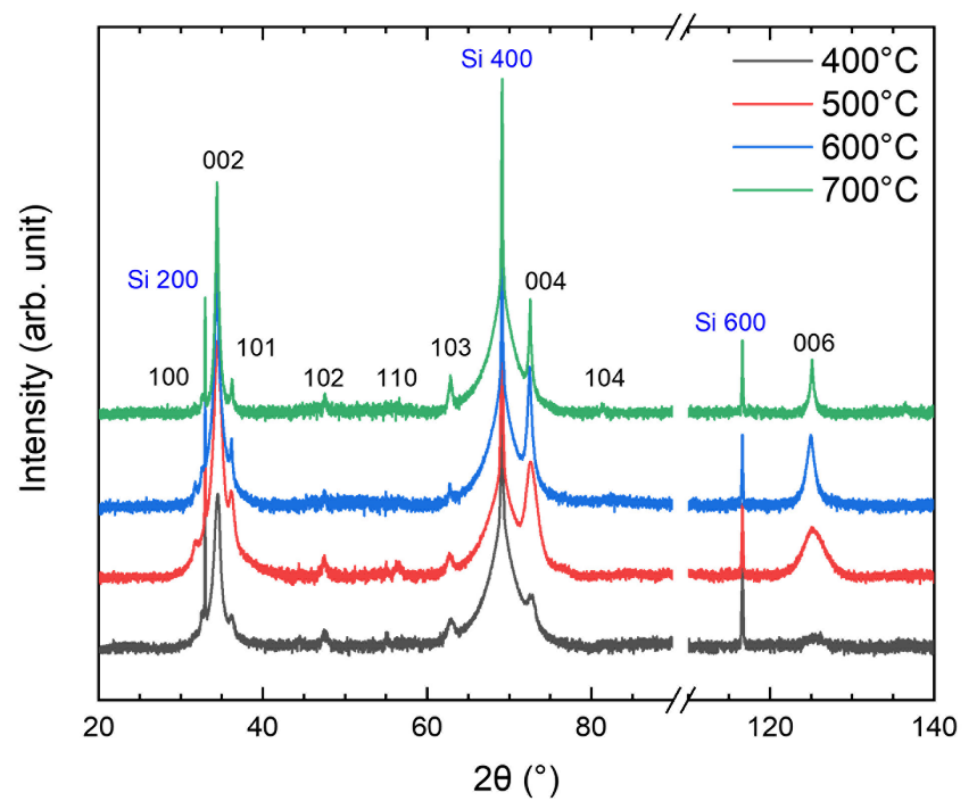

Figure 3. XRD patterns of $\mathrm{ZnO}$ deposits grown by PLI-MOCVD for a given growth temperature in the range of $400-700{ }^{\circ} \mathrm{C}$. The intensity is plotted in logarithm scale.

3.2. Growth texture and strain of $\mathrm{ZnO}$ deposits. $\mathrm{XRD}$ patterns of $\mathrm{ZnO}$ deposits grown by PLI-MOCVD are presented in Figure 3. Their analysis providing (002) texture coefficient, Williamson-Hall plot, $(00 l)$ crystallite size as well as homogeneous and inhomogeneous strains is represented in Figure 4. The Raman spectra of $\mathrm{ZnO}$ deposits grown by PLI-MOCVD are further presented in Figure 5.
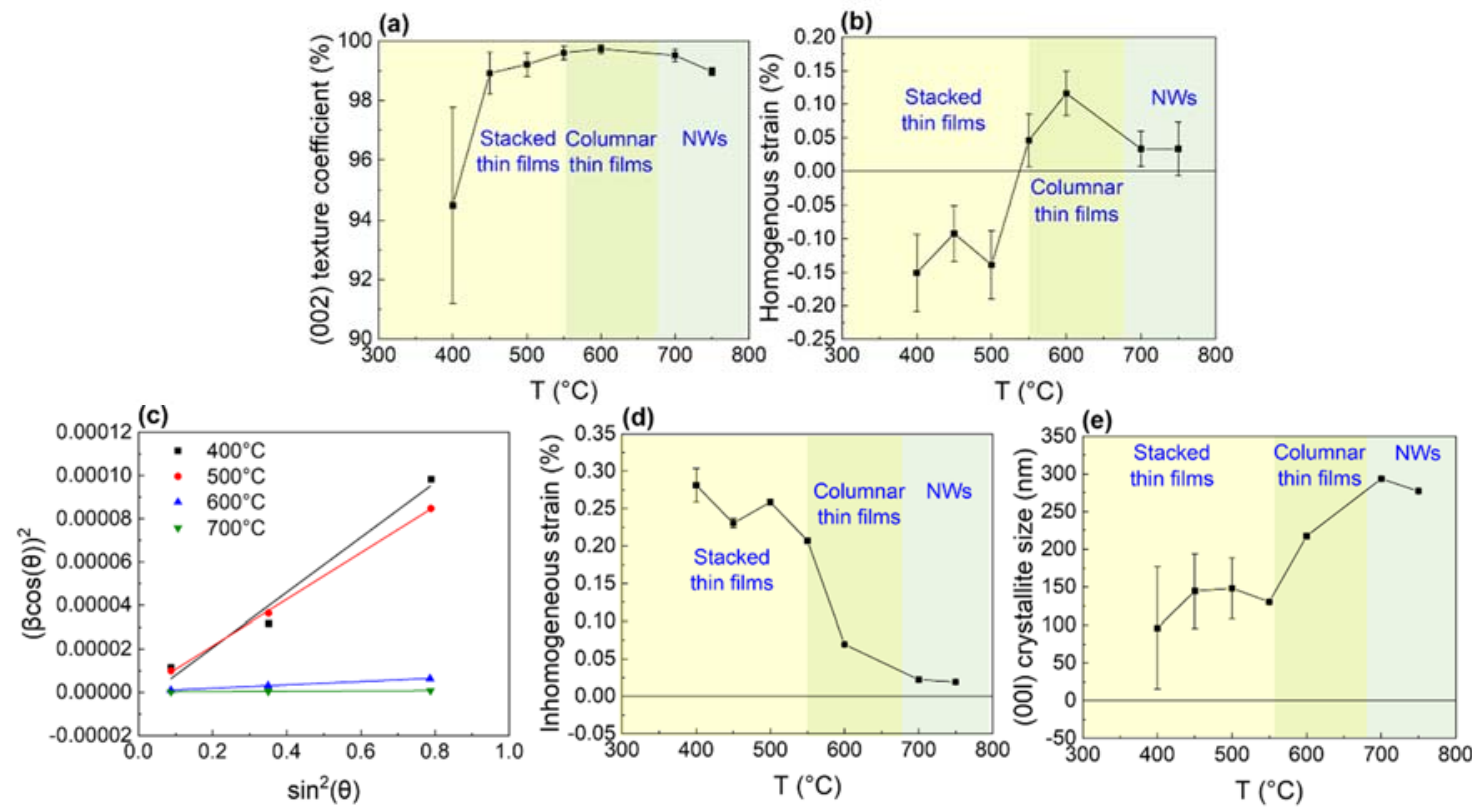
Figure 4. (a) Texture coefficient of the (002) diffraction peak vs growth temperature. (b) Homogeneous strain vs growth temperature. (c) Williamson-Hall plot of $\mathrm{ZnO}$ deposits grown by PLIMOCVD for a given growth temperature in the range of $400-700^{\circ} \mathrm{C}$. (d) Inhomogeneous strain vs growth temperature deduced from the Williamson-Hall plot. (e) (00l) crystallite size vs growth temperature inferred from the Williamson-Hall plot.

The XRD patterns of stacked thin films, columnar thin films, and nanowires show the typical diffraction peaks of the $\mathrm{ZnO}$ wurtzite structure, as indicated by the ICDD file labeled 00-036-1451. Correlatively, the Raman lines at 99 and $438 \mathrm{~cm}^{-1}$ are assigned to the $\mathrm{E}_{2}{ }^{\text {low }}$ and $\mathrm{E}_{2}{ }^{\text {high }}$ modes of the wurtzite structure, respectively. ${ }^{33}$ The drastic increase in their intensity by raising growth temperature from 400 to $750{ }^{\circ} \mathrm{C}$ specifically indicates a strong improvement of the crystallinity of $\mathrm{ZnO}$ deposits. Other Raman lines including the $\mathrm{A}_{1}(\mathrm{LO})$ and $\mathrm{E}_{1}(\mathrm{TO})$ modes are hardly detected owing to the low signal of $\mathrm{ZnO}$ deposits as compared to the signal of the $\mathrm{Si}$ substrate, even when the cross-polarization was used. The 002 diffraction peak pointing at $34.4^{\circ}$ has the highest intensity by several orders of magnitude as compared to the intensities of the $100,101,102,110,103,104$ diffraction peaks pointing at 31.8 , 36.3, 47.5, 56.6, 62.9, and $81.4^{\circ}$, respectively, as shown in Figure 3. This indicates that the $\mathrm{ZnO}$ deposits systematically grow along the polar $c$-axis. The texture coefficient of the 002 diffraction peak increases from 94.5 to $99.7 \%$, as growth temperature is raised from 400 to $600{ }^{\circ} \mathrm{C}$, as shown in Figure 4a. The transition from stacked to columnar thin films is thus associated with a more pronounced growth texture along the polar $c$-axis. More than $99 \%$ of grains are oriented along the polar $c$-axis. The texture coefficient of the 002 diffraction peak then decreases slightly to the value of $99.0 \%$ at the highest growth temperature of $750{ }^{\circ} \mathrm{C}$. The transition from columnar thin films to nanowires apparently deteriorates slightly the texture along the polar $c$-axis. This is due to the specific morphology of $\mathrm{ZnO}$ deposits grown at 700 and $750{ }^{\circ} \mathrm{C}$.

Additionally, the shift of the 002 diffraction peak as seen in Figure 3 corresponds to a deviation of the $c$ lattice parameter from its bulk value $c_{0}$ of $5.20661 \AA$. It was exploited to deduce the homogeneous strain $\varepsilon_{H S}$ in $\mathrm{ZnO}$ deposits using $\varepsilon_{H S}=c-c_{0} / c_{0}$, as revealed in Figure $\mathbf{4 b}$. The compressive homogeneous strain along the polar $c$-axis in stacked thin films gradually decreases from -0.15 to $-0.14 \%$ as growth temperature is raised from 400 to $500{ }^{\circ} \mathrm{C}$. Correlatively, the $\mathrm{E}_{2}^{\text {high }}$ line is shifted towards higher 
wavenumber. ${ }^{34}$ The compressive homogeneous strain originates from the Volmer-Weber growth mode of stacked thin films, in which the coalescence process of isolated islands at the onset of growth generates high biaxial tensile stresses. ${ }^{35}$ Interestingly, as growth temperature is further raised from 500 to $600{ }^{\circ} \mathrm{C}$, the homogeneous strain along the polar $c$-axis in columnar thin films is strongly relieved and progressively becomes tensile. By further raising growth temperature to $750^{\circ} \mathrm{C}$, the homogeneous strain gets the value close to zero in nanowires, as expected from the efficient strain relaxation on their lateral surfaces. ${ }^{36}$ Correlatively, the position of the $\mathrm{E}_{2}{ }^{\text {high }}$ line gets to the value of $438.3 \mathrm{~cm}^{-1}$, which is closer to the value of bulk $\mathrm{ZnO}$ at $438 \mathrm{~cm}^{-1}$. Additionally, the size (i.e. $\beta_{D}$ ) and strain (i.e. $\beta_{S}$ ) broadening of the 002, 004, and 006 diffraction peaks to their full-width-at-half-maximum $\beta$ were assessed within the Williamson-Hall plot by considering that the peaks are mainly described by a Gaussian function:

$$
\beta^{2}=\beta_{S}^{2}+\beta_{D}^{2}
$$

where $\beta_{S}=C \varepsilon_{I S} \tan \theta$ and $\beta_{D}=\frac{K \lambda}{D \cos \theta}$, for which $\theta$ is the Bragg angle, $C$ is a constant of $4,{ }^{37-38} K$ is a constant depending on the shape of the particles and typically equals to $0.9,{ }^{38} \lambda$ is the wavelength of the $\mathrm{K}_{\alpha 1}(\mathrm{Cu})$ source that is equal to $0.15406 \mathrm{~nm}, \varepsilon_{I S}$ is the inhomogeneous strain caused by the microstructural defects, and $L$ is the mean $(00 l)$ crystallite size. By rearranging Eq.(3), it follows:

$$
(\beta \cos \theta)^{2}=\left(C \varepsilon_{I S} \sin \theta\right)^{2}+\left(\frac{K \lambda}{L}\right)^{2}
$$

The Williamson-Hall plots in Figure 4c were extracted from the position and full-width-at-halfmaximum of the 002,004 , and 006 diffraction peaks in the XRD patterns of stacked thin films, columnar thin films, and nanowires, as presented in Figure 3. The inhomogeneous strain and mean $(00 l)$ crystallite size represented in Figure $4 \mathbf{d}$ and $\mathbf{4 e}$ were inferred from its slope and intercept using Eq.(4), respectively. ${ }^{37-38}$ The inhomogeneous strain coming from microstructural defects basically decreases from 0.28 to $0.01 \%$ as growth temperature is raised from 400 to $750{ }^{\circ} \mathrm{C}$. This corresponds to the transition from stacked thin films to nanowires, as expected from the gradual decrease in the density of extended defects in their center. The mean crystallite size of stacked thin films in the vertical direction progressively increases from 82 to $128 \mathrm{~nm}$ as growth temperature is raised from 400 to $550^{\circ} \mathrm{C}$. This is directly related to the increase in its thickness from 367 to $923 \mathrm{~nm}$. Eventually, it jumps from 128 to 277 
$\mathrm{nm}$, as the transition to columnar thin films and nanowires occur in the growth temperature range of 550 $-750{ }^{\circ} \mathrm{C}$. Moreover, two broad bands centered around 1360 and $1580-1600 \mathrm{~cm}^{-1}$ occur in the Raman spectra of all $\mathrm{ZnO}$ deposits, as seen in Figure 5. They can be assigned to $\mathrm{C}-\mathrm{C}$ bonds, originating from the residual organic molecules related to the by-products of DEZn chemical precursor and cyclohexane solvent. ${ }^{39}$

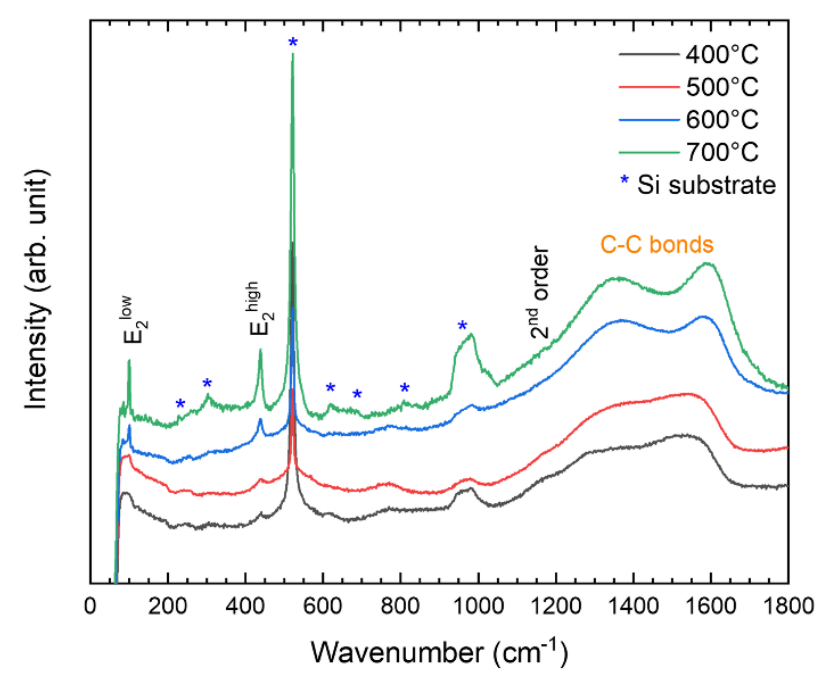

Figure 5. Raman spectra of $\mathrm{ZnO}$ deposits grown by PLI-MOCVD for a given growth temperature in the range of $400-700^{\circ} \mathrm{C}$.

3.3. Nucleation mechanisms of $\mathbf{Z n O}$ nanowires. The TEM image of $\mathrm{ZnO}$ deposits grown at $700{ }^{\circ} \mathrm{C}$ by PLI-MOCVD in Figure 6d-e indicates that the nucleation of nanowires is directly achieved on a very thin, amorphous, native $\mathrm{SiO}_{\mathrm{x}}$ layer without the formation of a continuous $\mathrm{ZnO}$ wetting layer. The direct nucleation of $\mathrm{ZnO}$ nanowires on $\mathrm{Si}$ substrates without using any seed/wetting layers is remarkable in the MOCVD process. It is definitely in contrast to the formation of $\mathrm{ZnO}$ nanowires by chemical bath deposition, requiring the typical use of a polycrystalline $\mathrm{ZnO}$ seed layer ${ }^{40}$ The transition from thin films to nanowires as growth temperature is raised was reported on (0001) sapphire substrate in $\operatorname{Ref}^{28}$, for which the nucleation and growth mechanisms were driven by the epitaxy between the $\mathrm{ZnO}$ deposits and sapphire. In that sense, the orientation of sapphire substrates have a strong influence on the morphology of $\mathrm{ZnO} .{ }^{41}$ Instead, no epitaxial relationship occurs in the present case, as shown in Figure 6e. At the bottom of $c$-axis oriented nanowires, a polycrystalline thin layer with a thickness of several tens of nanometers is mostly oriented along the polar $c$-axis, as revealed in the selected area diffraction 
(SAED) patterns in Figure 6c and by the HRTEM image of Figure 6e. It is basically formed prior to the formation of nanowires during growth. Following its thickening during growth, the development of vertical, elongated, $c$-axis oriented grains prevail over the grains oriented along other directions during a competitive process. This leads to the formation of isolated $c$-axis oriented nanowires with a high density, as shown in the SAED pattern in Figure $\mathbf{6 b}$ and by the TEM image of Figure 6a. The high growth temperature of 700 and $750{ }^{\circ} \mathrm{C}$ is required to form nanowires, by i) increasing the desorption of zinc and oxygen ad-atoms to create isolated grains and by ii) increasing the surface diffusion of zinc and oxygen ad-atoms along their sidewalls and hence enhancing their elongation along the $c$-axis to create isolated nanowires.
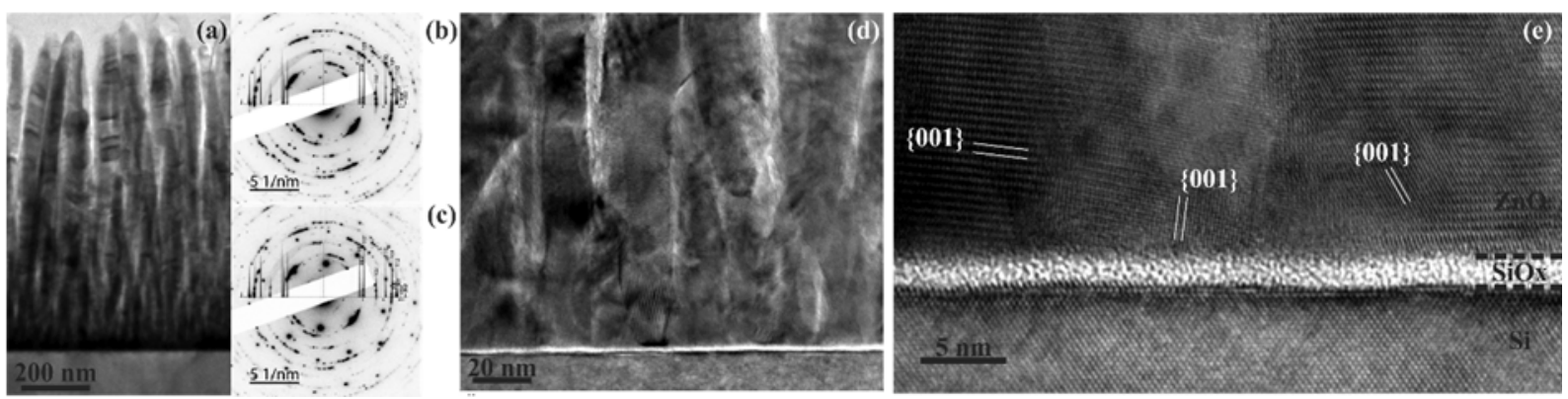

Figure 6. (a) Low magnification TEM image of nanowires grown at $700{ }^{\circ} \mathrm{C}$ by PLI-MOCVD. (b-c) SAED patterns recorded at the top and at the bottom of nanowires, respectively. (d-e) TEM and HRTEM images at the $\mathrm{ZnO} / \mathrm{Si}$ interface, respectively.

3.4. Optical properties of $\mathrm{ZnO}$ deposits. The spectroscopic properties of $\mathrm{ZnO}$ deposits grown by PLI-MOCVD as determined by CL measurements collected at $5 \mathrm{~K}$ are presented in Figure 7 . 


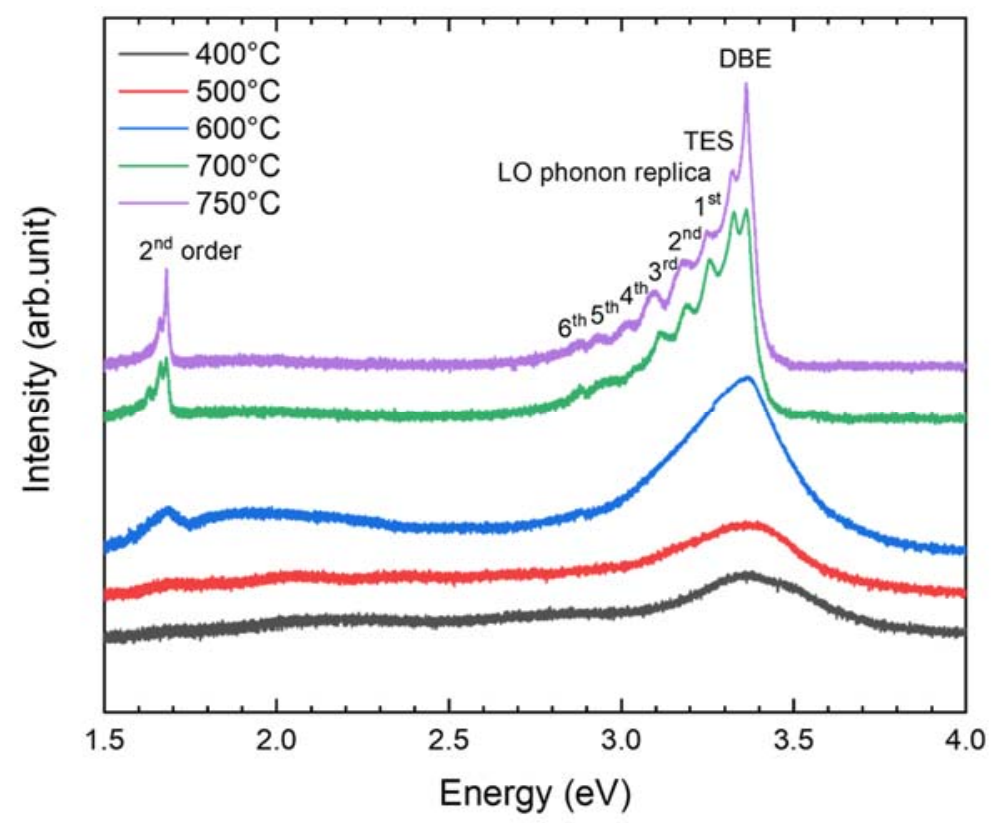

Figure 7. $5 \mathrm{~K} \mathrm{CL}$ spectra of $\mathrm{ZnO}$ deposits grown by PLI-MOCVD for a given growth temperature in the range of $400-750{ }^{\circ} \mathrm{C}$. The intensity is plotted in logarithm scale.

By raising growth temperature from 400 to $750{ }^{\circ} \mathrm{C}$, the near-band-edge (NBE) emissions switch from an asymmetric, broad band in the range of 3.0 to $3.7 \mathrm{eV}$ in stacked and columnar thin films to a highly structured, sharp band in nanowires. Correlatively, the CL intensity of the NBE emission drastically increases by a factor of about 650 in nanowires. This indicates again the strong improvement of the crystallinity and also of the optical quality of $\mathrm{ZnO}$ deposits by raising the growth temperature, which is in agreement with XRD and Raman scattering measurements. The NBE emission of nanowires is dominated by the line at $3.362 \mathrm{eV}$, which is attributed to radiative transitions involving neutral donorbound A-excitons (DBE). In the I nomenclature, ${ }^{42-43}$ the present radiative transitions may be assigned to the $\mathrm{I}_{6}$ line involving the presence of $\mathrm{Al}$ substituting for zinc sites $\left(\mathrm{Al}_{\mathrm{Zn}}\right)$ and acting as shallow residual donors with an ionization energy of $51.5 \mathrm{meV}$. It may also be due to the $Z_{3}$ and $Z_{4}$ lines, which are commonly associated with the incorporation of carbon species. ${ }^{44}$ No sign for the presence of Raman lines associated with hydrogen-related defects occurs, excluding the roles of the $\mathrm{I}_{4}{ }^{45}$ and $\mathrm{I}_{5}{ }^{46}$ lines as in $\mathrm{ZnO}$ nanowires grown by chemical bath deposition. ${ }^{47}$ Instead, the formation of $\mathrm{Al}_{\mathrm{Zn}}$ is expected to be due to the residual contamination of the MOCVD system while the incorporation of carbon species may come from the decomposition of the chemical precursors. Additionally, an intense line located at 3.326 $\mathrm{eV}$ is attributed to radiative transitions usually involving two-electron satellites (TES). The present line 
may be assigned, to some extent, to TES in the $2 \mathrm{~s}$ state involving $\mathrm{Al}_{\mathrm{Zn}}$ or in the $2 \mathrm{p}$ state involving the $\mathrm{Z}_{3}$ line. However, the significant shift from their theoretical position around $3.323 \mathrm{eV}$ certainly accounts for the occurrence of additional contributions. Interestingly, the TEM and HRTEM images in Figure 8 clearly reveal the presence of basal-plane stacking faults (BSFs) in the center of nanowires. They are basically identified as being of $\mathrm{I}_{1}$-type with the plane stacking sequence ...ABABABCACACA.... ${ }^{48}$ This type of BSF is typically not related to strain relaxation, but nucleates during growth owing to its low formation energy. Importantly, the $\mathrm{I}_{1}$-type BSFs are expected to form a quantum-well-like structure that can confine excitons and induce an emission around $3.321 \mathrm{eV}$ in $\mathrm{ZnO}$ thin films and $3.329 \mathrm{eV}$ in $\mathrm{ZnO}$ nanowires, as reported in Refs. ${ }^{49-50}$, respectively. As a consequence, both the TES line (i.e. $\mathrm{Al}_{\mathrm{Zn}}$ and carbon species) at $3.323 \mathrm{eV}$ and BSF line (i.e. $\mathrm{I}_{1}$ ) at $3.329 \mathrm{eV}$ contribute to the intense line located at the intermediate energy of $3.326 \mathrm{eV}$.

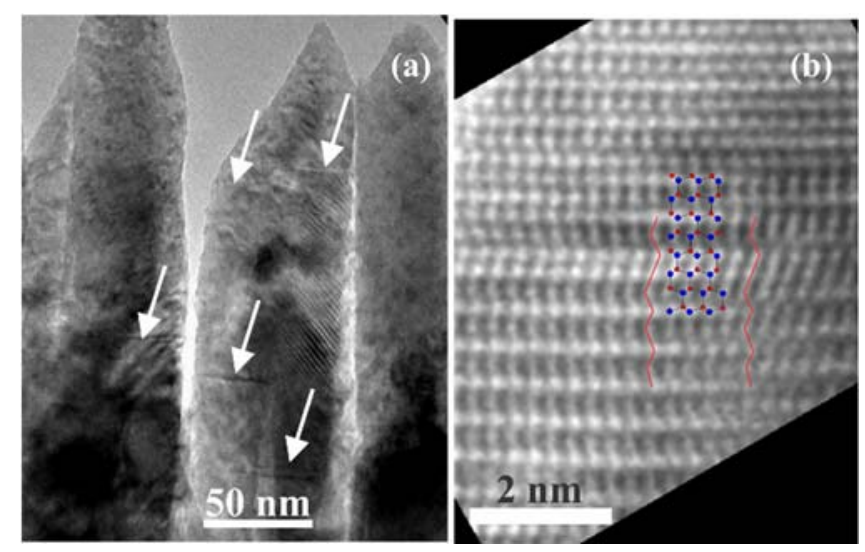

Figure 8. (a) Low-magnification TEM image of $\mathrm{ZnO}$ nanowires grown at $700{ }^{\circ} \mathrm{C}$ by PLI-MOCVD. The arrows indicate the presence of BSFs (b) HRTEM image where the stacking sequences and atomic model of an $\mathrm{I}_{1}$-type stacking fault have been superimposed.

The 3.259, 3.187, 3.116, 3.042, 2.956, and $2.879 \mathrm{eV}$ lines are further associated with first, second, third, fourth, fifth, and sixth longitudinal-optical (LO) phonon replica of the TES and BSF lines, respectively. Each of them is roughly separated by an energy of $72 \mathrm{meV}$, which basically corresponds to the phonon energy in $\mathrm{ZnO}$. The intensity of these LO phonon replica basically follows the Poisson distribution: ${ }^{43}$

$$
I_{n}=\frac{e^{-S} \cdot S^{n}}{n !} I_{0}
$$


where $n$ is the number of the LO phonon replica, $I_{0}$ is the intensity of the zero-phonon TES/BSF lines, $I_{n}$ is intensity of the $\mathrm{n}^{\text {th }}$ LO phonon replica, and $S$ is the Huang-Rhys constant assessing the coupling strength of the electronic transition to the LO polarization field (i.e. phonon coupling). By taking into account the ratios of $I_{n}$ over $I_{0}$ in nanowires as plotted in Figures S6a-b, the $S$ values of about 0.8 are deduced from the fitting with Eq. (5). The present value is much larger than the typical value of the TES line related to $\mathrm{Al}_{\mathrm{Zn}}$ that is equal to 0.058 , as reported in $\mathrm{Ref}^{43}$. This indicates a stronger phonon coupling in nanowires, which is consistent with the additional contribution of the BSF line on which the localization may be enhanced.

In the range of deeper emissions corresponding to the visible band lying from $2-3 \mathrm{eV}$, several minor, broad peaks occur in stacked and columnar thin films, but with an intensity about one order of magnitude smaller than the intensity of the NBE emission. In contrast, no significant visible band occurs in nanowires, indicating again an improvement of the optical quality. ${ }^{28}$ From the most recent theoretical calculations, ${ }^{51-52}$ the peaks in the visible band originates mainly from the complexes involving zinc vacancy with hydrogen and/or residual donors. Their absence again excludes the significant role of hydrogen-related defects in the present nanowires.

3.5. Piezoelectric properties and polarity of $\mathrm{ZnO}$ deposits. The tunable morphology of $\mathrm{ZnO}$ deposits grown by PLI-MOCVD in the same system by only changing the growth temperature offers a great, unique opportunity to investigate the piezoelectric properties of $\mathrm{ZnO}$ and specifically its dependence on its geometry and dimensions. In other words, by using the same MOCVD system and by fixing most of the growth conditions (e.g. total pressure, flow rate, and nature of chemical precursors), we can decouple geometrical and dimensional effects from other electrical effects on the piezoelectric properties of $\mathrm{ZnO}$ deposits. This is basically not possible when comparing the experimental data collected on $\mathrm{ZnO}$ deposits grown by different deposition techniques. The topography of a $1 \mu \mathrm{m}^{2}$-area on the stacked thin film, columnar thin film, and nanowires along with their correlated piezoelectric amplitude and phase as measured by DataCube PFM are presented in Figure 9. The corresponding histograms are shown in Figure 10. 


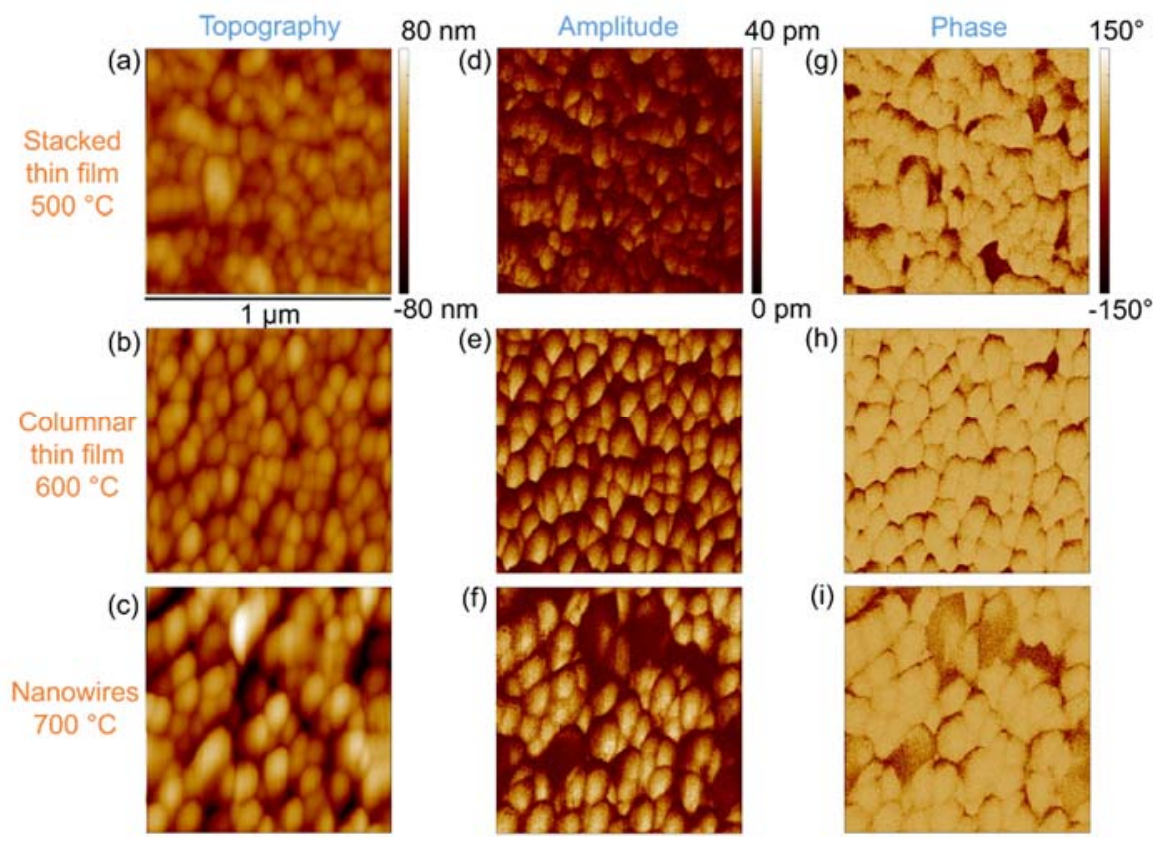

Figure 9. Topography of $\mathrm{ZnO}$ deposits grown at (a) $500{ }^{\circ} \mathrm{C}$, (b) $600{ }^{\circ} \mathrm{C}$ and (c) $700{ }^{\circ} \mathrm{C}$; piezoelectric amplitude of $\mathrm{ZnO}$ deposits grown at (d) $500{ }^{\circ} \mathrm{C}$ (e) $600{ }^{\circ} \mathrm{C}$ and (f) $700{ }^{\circ} \mathrm{C}$; piezoelectric phase of $\mathrm{ZnO}$ deposits grown at (g) $500{ }^{\circ} \mathrm{C}$ (h) $600{ }^{\circ} \mathrm{C}$ and (i) $700{ }^{\circ} \mathrm{C}$.

Figures 9d-f show an inhomogeneous piezoelectric amplitude on the surface of the three $\mathrm{ZnO}$ deposits, varying from 0 to $35 \mathrm{pm}$ when an $\mathrm{AC}$ drive voltage of $5 \mathrm{~V}$ was applied. As a result, the effective piezoelectric coefficient is estimated to be in the range of 0 to $7 \mathrm{pm} / \mathrm{V}$, which is lower than the values reported in Ref. ${ }^{1}$. We expect that the highly-doped Si substrate used as the bottom electrode could lead to significant losses. Accordingly, the actual voltage applied on the $\mathrm{ZnO}$ deposits could be smaller than $5 \mathrm{~V}$, resulting in an underestimation of the piezoelectric coefficient. Among the three deposits, the amplitude image of the stacked thin film is clearly darker as compared to the columnar thin film and nanowires. This shows that its amplitude response is significantly lower. Only a very few domains reach an amplitude of $20 \mathrm{pm}$ on the stacked thin film, while most of the domains on the columnar thin film can have an amplitude around this value. In the nanowire deposit, some domains present nearly no amplitude response, which could be due to the remaining PMMA after etching (see Figure S4). The amplitude on the nanowires is also slightly higher than the amplitude on the columnar thin film. Their histograms in Figures 10a-c show the shift of the amplitude distribution towards higher values when the morphology evolves from the stacked thin film to nanowires through the columnar thin film. The enhancement of the piezoelectric response can be correlated with the structural analysis presented above, 
where the transition from stacked thin films to nanowires is accompanied by a more pronounced growth texture along the polar $c$-axis along with a better crystallinity.
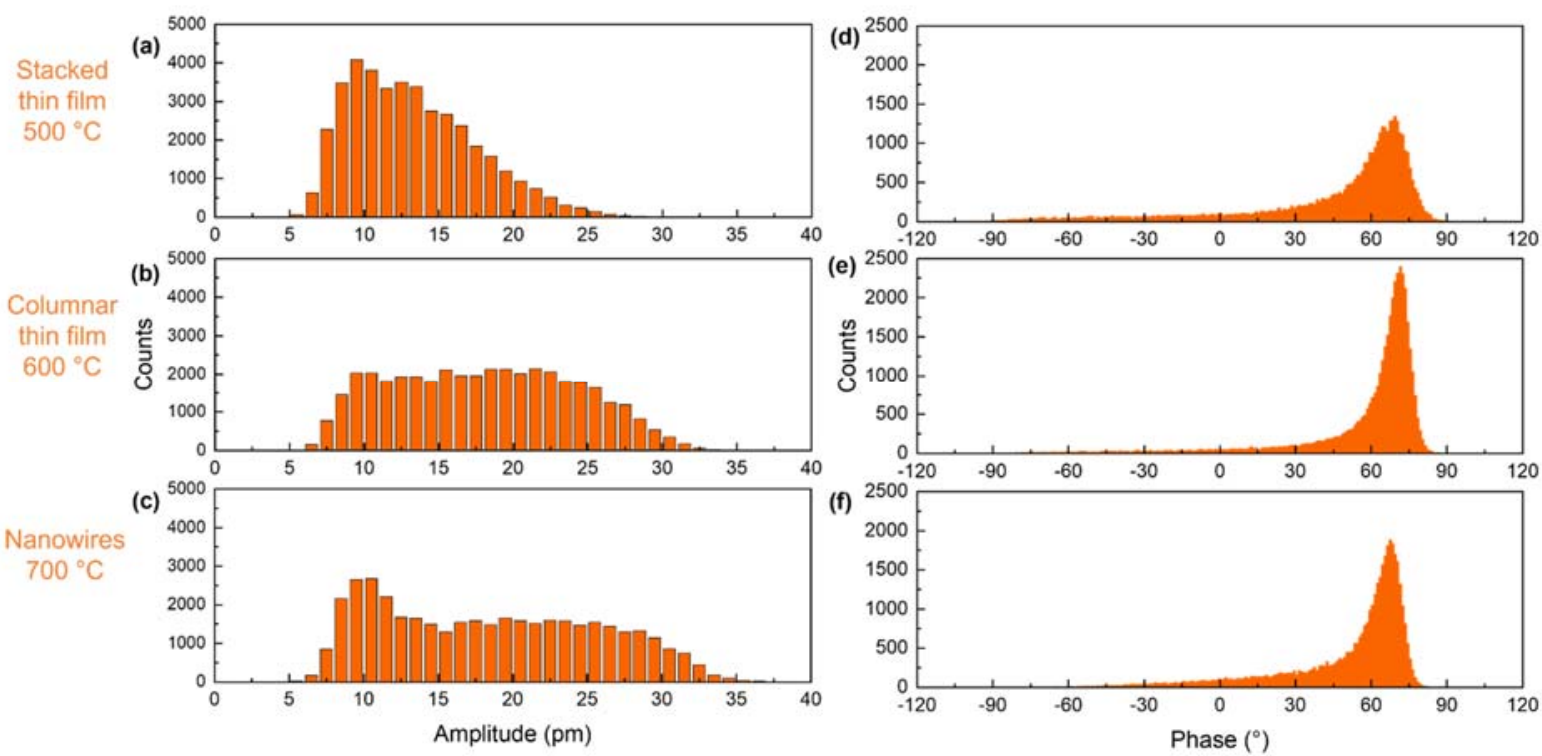

Figure 10. Raw piezoelectric amplitude histograms of $\mathrm{ZnO}$ deposits grown at (a) $500^{\circ} \mathrm{C}$, (b) $600^{\circ} \mathrm{C}$ and (c) $700^{\circ} \mathrm{C}$. Raw piezoelectric phase histograms of $\mathrm{ZnO}$ deposits grown at (d) $500^{\circ} \mathrm{C}$, (e) $600^{\circ} \mathrm{C}$ and (f) $700^{\circ} \mathrm{C}$.

The PFM measurements also show notable results concerning the piezoelectric phase, as shown in Figures 9g-i. On these images, the domains with the phase values around $70^{\circ}$ (i.e. bright domains) and $-90^{\circ}$ (i.e. dark domains) are attributed to the $\mathrm{Zn}$ - and O-polarity, respectively (see Figures $\mathbf{S 3}$ and S7 of Supporting Information). Both domains with either polarity can be seen on the stacked thin film in Figure 9g, demonstrating the coexistence of $\mathrm{Zn}$ - and O-polarity. In contrast to the stacked thin film, almost all the domains of the columnar thin film are Zn-polar, as presented in Figure $\mathbf{9 h}$. In the case of nanowires, most of the domains are also Zn-polar, as revealed in Figure 9i, while the dark domains originate from the remaining PMMA (see Figure S4). Correspondingly, the peaks at $70^{\circ}$ attributed to Zn-polar domains are clearly observed in the phase histograms of these deposits, as shown in Figures 10d-f. The small signals related to O-polar domains are seen in the phase histogram of the stacked thin film, while being negligible in the columnar thin film and nanowires. Besides, other signals distributed in all the range from $-180^{\circ}$ to $180^{\circ}$ in these phase histograms are due to noise coming from nonpiezoelectric signals (see Figure S7 of Supporting Information). The non-piezoelectric signals at grain 
boundaries on the thin film deposits or on the PMMA deposited on top of the nanowires also contribute to the amplitude histograms (see Figures 10a-c), which cause a small inaccurate estimation of amplitude, specifically in the case of nanowires. Further PFM data treatments were performed to remove the data points of these non-piezoelectric domains and separate the amplitude response of O- and $\mathrm{Zn}$ polar data points in order to have more accurate comparison of the piezoelectric responses among the $\mathrm{ZnO}$ deposits, as shown in Figure 11. Moreover, the DataCube PFM measurements were also performed on different regions of the deposits and confirmed with classical PFM measurements.
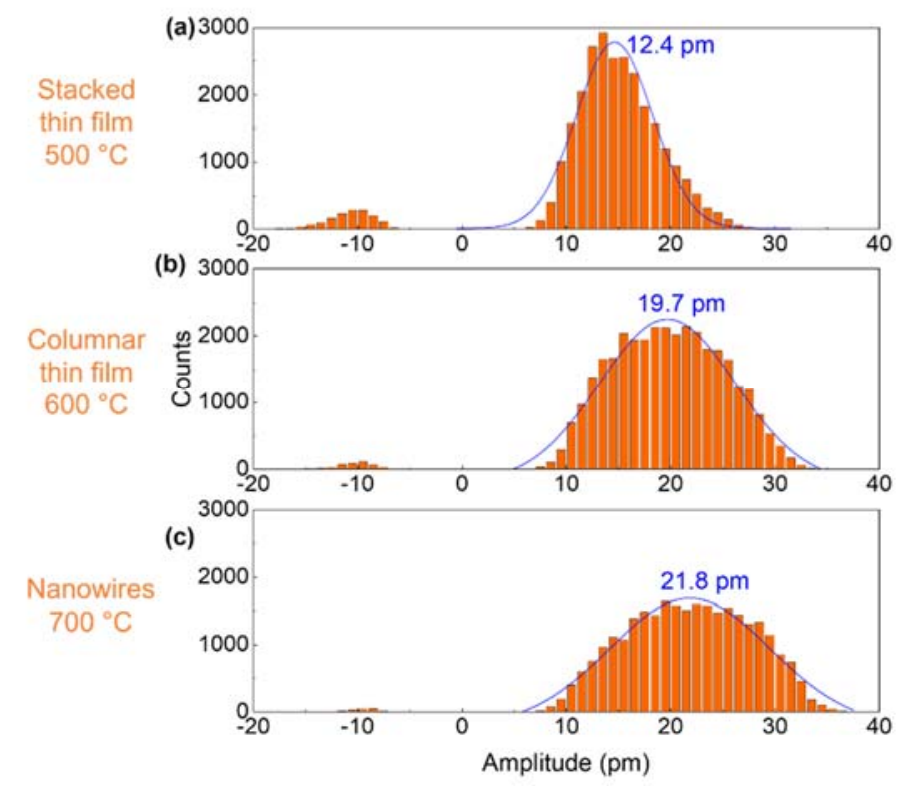

Figure 11. Piezoelectric amplitude histograms of $\mathrm{ZnO}$ deposits grown at (a) $500^{\circ} \mathrm{C}$, (b) $600^{\circ} \mathrm{C}$ and (c) $700^{\circ} \mathrm{C}$ following the data treatments where data points with the phase response higher than $50^{\circ}$ were kept without modification (representing the Zn-polar facet), while data points with the phase response lower than $-50^{\circ}$ had their amplitude value multiplied by -1 (representing the O-polar facet). Other data points were removed from the histograms.

Overall, the results show that the amplitude response of the Zn-polar domains increases from $12.4 \mathrm{pm}$ to $21.8 \mathrm{pm}$ when the $\mathrm{ZnO}$ morphology changed from stacked thin film to nanowires, as shown in Figure 11. In contrast, the amplitude response of the O-polar domains keeps a constant value around $-10 \mathrm{pm}$. Notably, the proportion of $\mathrm{Zn}$-polar domains also reaches nearly $100 \%$ in the columnar thin film and nanowires (see Figure S8 of Supporting Information). As the Zn- and O-polar domains are opposite in polarity, the existence of both polarities on the stacked thin film can induce the cancellation of the overall 
piezoelectric output when integrated into devices with top and bottom electrodes. The significant prevalence of the Zn-polar domains on the columnar thin film and nanowires can lead to the higher piezoelectric output on these deposits as compared to the stacked thin film. Furthermore, it is revealed in Figure S9 of the Supporting Information that the electrical resistivity as deduced from TLM method on the stacked thin film and columnar thin film is very similar and of about $6.5 \Omega . \mathrm{cm}$. This indicates that the increase in the piezoelectric amplitude from $12.4 \mathrm{pm}$ on the stacked thin film to $19.7 \mathrm{pm}$ on the columnar thin film is mostly related to the change of morphology by forming elongated columnar $\mathrm{Zn}$ polar grains crossing the entire thickness. Similarly, the further increase in the piezoelectric amplitude to $21.8 \mathrm{pm}$ on the nanowires is associated with the formation of high aspect ratio $\mathrm{Zn}$-polar nanostructures exhibiting a high surface over volume ratio.

\section{DISCUSSION}

The morphology transition of $\mathrm{ZnO}$ deposits grown by PLI-MOCVD by only tuning the growth temperature is summarized in Figure 12. The morphology transitions are thermally activated and thus driven by thermodynamic and kinetic considerations. The transition from the stacked to columnar thin films is relatively typical in polycrystals when the growth temperature is raised ${ }^{35}$ and here benefits from the strong anisotropy of the wurtzite structure. The formation of isolated nanowires develops the surface area of the non-polar $m$-plane sidewalls with the lowest surface energy in the wurtzite structure. ${ }^{53-54}$ This results in the minimization of the free energy of the system as the driving force for the morphology transition. The increase in the growth temperature also enhances i) the desorption of zinc and oxygen ad-atoms to form isolated nanowires and ii) their surface diffusion along the sidewalls to increase the nanowire aspect ratio. ${ }^{55}$ The possibility to tune the morphology of $\mathrm{ZnO}$ deposits is of great interest for many nanoscale devices including piezoelectric devices, ${ }^{56}$ LEDs, and solar cells, ${ }^{57-58}$ where the geometrical and dimensional constraints play a significant role on their performances. In particular, it is shown here that the morphology of $\mathrm{ZnO}$ nanowires boosts the piezoelectric amplitude with respect to the stacked and columnar thin films. This is attributed to several phenomena occurring in the $\mathrm{ZnO}$ nanowires. First, the higher crystalline quality and larger growth texture along the piezoelectric $c$-axis in nanowires is highly favorable. The increase in the number of domains oriented along the piezoelectric 
$c$-axis inherently results in an increase in the piezoelectric amplitude. Second, the improvement of the polarity uniformity has a significant impact, specifically the predominance of the Zn-polarity over the O-polarity to avoid any cancellation of the piezoelectric amplitude. The polarity issue in $\mathrm{ZnO}$ is of great significance for piezoelectric devices, but surprisingly it has not been investigated in details yet. The formation of $\mathrm{Zn}$-polar $\mathrm{ZnO}$ nanowires using PLI-MOCVD is in general agreement with the literature reporting that $\mathrm{ZnO}$ nanowires spontaneously grown by vapor phase techniques are systematically $\mathrm{Zn}$ polar, ${ }^{59-60}$ while $\mathrm{O}$ - and $\mathrm{Zn}$-polar $\mathrm{ZnO}$ nanowires can be formed by chemical bath deposition. ${ }^{61}$ Additionally, the Zn-polar domains are found here to exhibit a larger piezoelectric amplitude than the O-polar domains. This may be attributed to the incorporation of a smaller amount of residual impurities (i.e. $\mathrm{Al}_{\mathrm{Zn}}$ ) in $\mathrm{Zn}$-polar $\mathrm{ZnO}$ nanowires, leading to a decrease in the density of free charge carriers and thus to a reduction of the screening potential. The present differential incorporation has been reported in the chemical vapor deposition of $\mathrm{ZnO}$ thin films with either polarity. ${ }^{62}$ It may be opposite to what is commonly reported in the case of $\mathrm{O}$ - and $\mathrm{Zn}$-polar $\mathrm{ZnO}$ nanowires grown by chemical bath deposition. ${ }^{47}$ Third, the geometrical constraint related to high aspect ratio nanostructures is of high potential. The large surface area involving the non-polar $m$-plane sidewalls and top-c-faces can give rise to an efficient Fermi Level surface pinning. This can somehow deplete the objects and hence limit the screening potential by the free charge carriers. ${ }^{63}$ Overall, the present findings show the strong interest in developing $\mathrm{ZnO}$ nanowires by PLI-MOCVD for piezoelectric applications and emphasize the importance of controlling the nature of the polarity and its uniformity to enhance the performances of the related devices.

\section{Growth temperature}

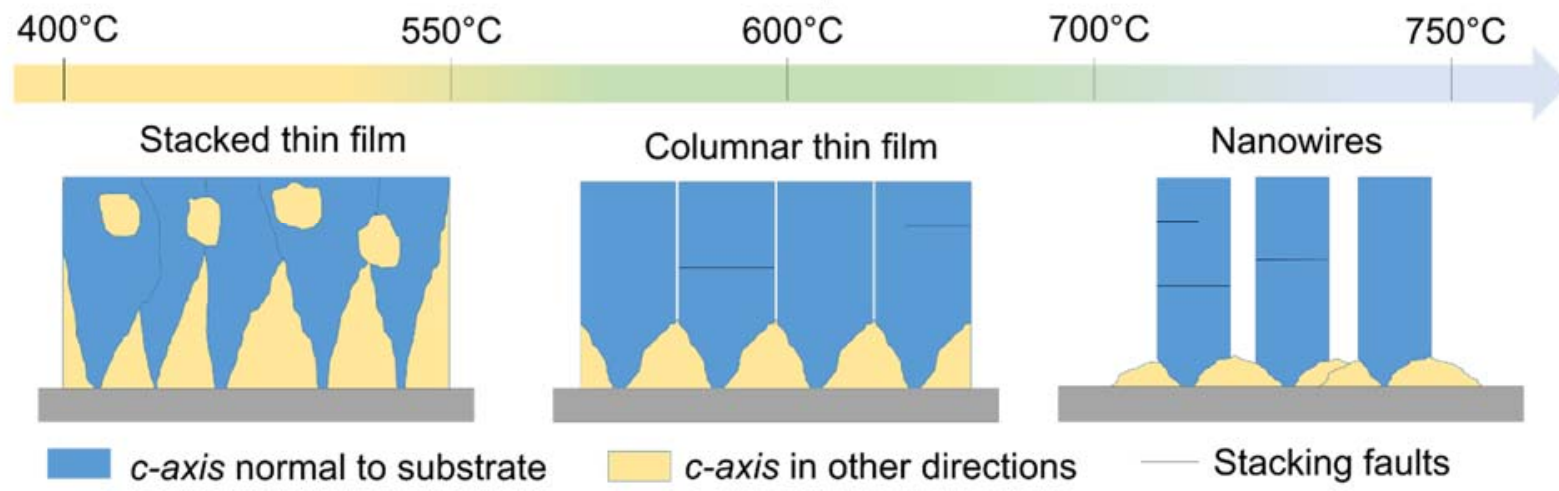


Figure 12. Schematic of the formation mechanisms of $\mathrm{ZnO}$ deposits grown by PLI-MOCVD for a given growth temperature in the range of $400-750^{\circ} \mathrm{C}$.

\section{CONCLUSION}

In summary, the decoupling of the effects of the morphology, dimensions, and electrical properties of $\mathrm{ZnO}$ deposits on their piezoelectric properties is still open to a large extent. By using PLI-MOCVD, we have shown the morphology transition of $\mathrm{ZnO}$ deposits on silicon from stacked thin films to nanowires through columnar thin films by only varying the growth temperature from 400 to $750{ }^{\circ} \mathrm{C}$. A detailed analysis of their formation mechanisms has further been revealed with a complete growth diagram. The morphology transition results in a strong enhancement of the crystallinity of $\mathrm{ZnO}$ deposits and of their growth texture along the $c$-axis, together with a massive relaxation of the strain in nanowires. It further leads to a prevailed zinc polarity, for which its uniformity is greatly enhanced in nanowires. $\mathrm{I}_{1}$-type basal-plane stacking faults are further found to be formed in nanowires and contribute to an emission line around $3.326 \mathrm{eV}$ with a fairly low phonon coupling in cathodoluminescence. Interestingly, the transition additionally leads to a significant increase in the piezoelectric amplitude, as determined by Datacube PFM measurements, while the electrical resistivity of $\mathrm{ZnO}$ deposits is very similar. The $\mathrm{Zn}$ polar domains additionally exhibit a larger piezoelectric amplitude than the O-polar domains, indicating the importance of controlling the nature of the polarity in $\mathrm{ZnO}$ deposits to improve the performances of piezoelectric devices. These findings directly demonstrate the impact of the structural properties of $\mathrm{ZnO}$ deposits in terms of morphology, growth texture, and polarity on their piezoelectric properties and show the high potential in using the PLI-MOCVD system for the present field. They further reveal unambiguously the superiority of nanowires over thin films for piezoelectric devices.

\section{ASSOCIATED CONTENT}

Supporting Information Available: Further experimental observations and results on the $\mathrm{ZnO}$ deposits; piezoelectric amplitude and phase response versus drive frequency (Figure S1 and S2); PFM measurements on two opposite polarity domains of periodically-poled lithium niobate (PPLN) using two different tips (Figure S3); SEM images of PMMA covered nanowires after etching for different 
times (Figure S4); series of metallic electrodes deposited on a thin film surface (Figure S5); ratio of LO phonon replica intensities to zero-phonon TES/BSF line intensity on nanowires (Figure S6); PFM measurements on $\mathrm{ZnO}$ single crystals with identified polarities and on sapphire (Figure S7); influence of the growth temperature on the piezoelectric amplitude and percentage pf Zn-polar domains (Figure S8); total resistance versus distance in TLM (Figure S9).

\section{ACKNOWLEDGEMENTS}

This work was supported by the French National Research Agency in the framework of the "Investissements d'avenir" program (ANR-15-IDEX-02) through the project CDP NEED. Q.C.B. held a doctoral fellowship from the project CDP NEED. The authors further acknowledge the support from the CNRS Renatech Network through the "Plateforme Technologique Amont" in a cleanroom environment. This research has also benefited from some of the characterization equipments of the Grenoble INP-CMTC platform. The authors are further grateful to Thomas Cossuet and Elea Matheret, Grenoble, France, for their assistance in the MOCVD system and preparation of the TEM lamellas, respectively.

\section{REFERENCES}

1. Ozgur, U.; Alivov, Y. I.; Liu, C.; Teke, A.; Reshchikov, M. A.; Dogan, S.; Avrutin, V.; Cho, S. J.; Morkoc, H. A Comprehensive Review of ZnO Materials and Devices. J. Appl. Phys. 2005, 98, 103.

2. Kolodziejczak-Radzimska, A.; Jesionowski, T. Zinc Oxide-from Synthesis to Application: A Review. Materials 2014, 7, 2833-2881.

3. Willander, M., Nur, O.; Zhao, Q. X.; Yang, L. L.; Lorenz, M.; Cao, B. Q.; Zuniga-Perez, J.; Czekalla, C.; Zimmermann, G.; Grundmann, M.; Bakin, A.; Behrends, A.; Al-Suleiman, M.; El-Shaer, A.; Che Mofor, A.; Postels, B.; Waag, A.; Boukos, N.; Travlos, A.; Kwack, H. S.; Guinard, J.; Le Si Dang, D. Zinc Oxide Nanorod Based Photonic Devices: Recent Progress in Growth, Light Emitting Diodes and Lasers. Nanotechnology 2009, 20, 40.

4. Consonni, V.; Briscoe, J.; Karber, E.; Li, X.; Cossuet, T. ZnO Nanowires for Solar Cells: A Comprehensive Review. Nanotechnology 2019, 30, 41.

5. Tian, W.; Lu, H.; Li, L. Nanoscale Ultraviolet Photodetectors Based on One-Dimensional Metal Oxide Nanostructures. Nano Res. 2015, 8, 382-405.

6. Wang, Z. L. Towards Self-Powered Nanosystems: From Nanogenerators to Nanopiezotronics. Adv. Funct. Mater. 2008, 18, 3553-3567.

7. Briscoe, J.; Dunn, S. Piezoelectric Nanogenerators - a Review of Nanostructured Piezoelectric Energy Harvesters. Nano Energy 2015, 14, 15-29.

8. Tao, R.; Parmar, M.; Ardila, G.; Oliveira, P.; Marques, D.; Montes, L.; Mouis, M. Performance of ZnO Based Piezo-Generators under Controlled Compression. Semicond. Sci. Technol. 2017, 32, 10. 
9. Parmar, M.; Perez, E.; Ardila, G.; Saoutieff, E.; Pauliac-Vaujour, E.; Mouis, M. A Demonstration of the Mechanical Sensing Capability of Individually Contacted Vertical Piezoelectric Nanowires Arranged in Matrices. Nano Energy 2019, 56, 859-867.

10. Yao, B. D.; Chan, Y. F.; Wang, N. Formation of ZnO Nanostructures by a Simple Way of Thermal Evaporation. Appl. Phys. Lett. 2002, 81, 757-759.

11. Carcia, P. F.; McLean, R. S.; Reilly, M. H.; Nunes, G. Transparent ZnO Thin-Film Transistor Fabricated by Rf Magnetron Sputtering. Appl. Phys. Lett. 2003, 82, 1117-1119.

12. Kaidashev, E. M.; Lorenz, M.; von Wenckstern, H.; Rahm, A.; Semmelhack, H. C.; Han, K. H.; Benndorf, G.; Bundesmann, C.; Hochmuth, H.; Grundmann, M. High Electron Mobility of Epitaxial ZnO Thin Films on C-Plane Sapphire Grown by Multistep Pulsed-Laser Deposition. Appl. Phys. Lett. 2003, 82, 3901-3903.

13. Chen, Y. F.; Bagnall, D. M.; Zhu, Z. Q.; Sekiuchi, T.; Park, K. T.; Hiraga, K.; Yao, T.; Koyama, S.; Shen, M. Y.; Goto, T. Growth of ZnO Single Crystal Thin Films on C-Plane $\left(\begin{array}{llll}0 & 0 & 1\end{array}\right)$ Sapphire by Plasma Enhanced Molecular Beam Epitaxy. J. Cryst. Growth 1997, 181, 165-169.

14. Natsume, Y.; Sakata, H. Zinc Oxide Films Prepared by Sol-Gel Spin-Coating. Thin Solid Films 2000, 372, 30-36.

15. Vayssieres, L. Growth of Arrayed Nanorods and Nanowires of $\mathrm{ZnO}$ from Aqueous Solutions. Adv. Mater. 2003, 15, 464-466.

16. Goux, A.; Pauporte, T.; Chivot, J.; Lincot, D. Temperature Effects on ZnO Electrodeposition. Electrochim. Acta 2005, 50, 2239-2248.

17. Carcia, P. F.; McLean, R. S.; Reilly, M. H. High-Performance ZnO Thin-Film Transistors on Gate Dielectrics Grown by Atomic Layer Deposition. Appl. Phys. Lett. 2006, 88, 3.

18. Liu, Y.; Gorla, C. R.; Liang, S.; Emanetoglu, N.; Lu, Y.; Shen, H.; Wraback, M. Ultraviolet Detectors Based on Epitaxial ZnO Films Grown by MOCVD. J. Electron. Mater. 2000, 29, 69-74.

19. Lee, W.; Jeong, M. C.; Myoung, J. M. Catalyst-Free Growth of ZnO Nanowires by Metal-Organic Chemical Vapour Deposition (MOCVD) and Thermal Evaporation. Acta Mater. 2004, 52, 3949-3957.

20. Park, W. I.; Yi, G. C.; Kim, M. Y.; Pennycook, S. J. ZnO Nanoneedles Grown Vertically on Si Substrates by Non-Catalytic Vapor-Phase Epitaxy. Adv. Mater. 2002, 14, 1841-1843.

21. Zhang, B. P.; Wakatsuki, K.; Binh, N. T.; Usami, N.; Segawa, Y. Effects of Growth Temperature on the Characteristics of ZnO Epitaxial Films Deposited by Metalorganic Chemical Vapor Deposition. Thin Solid Films 2004, 449, 12-19.

22. Montenegro, D. N.; Souissi, A.; Martinez-Tomas, C.; Munoz-Sanjose, V.; Sallet, V. Morphology Transitions in ZnO Nanorods Grown by MOCVD. J. Cryst. Growth 2012, 359, 122-128.

23. Sallet, V.; Thiandoume, C.; Rommeluere, J. F.; Lusson, A.; Riviere, A.; Riviere, J. P.; Gorochov, O.; Triboulet, R.; Munoz-Sanjose, V. Some Aspects of the MOCVD Growth of ZnO on Sapphire Using Tert-Butanol. Mater. Lett. 2002, 53, 126-131.

24. Scalisi, A. A.; Toro, R. G.; Malandrino, G.; Fragala, M. E.; Pezzotti, G. Growth of ZnO Nanostructures Produced by MOCVD: A Study of the Effect of the Substrate. Chem. Vapor Depos. 2008, 14, 115-122.

25. Bang, K. H.; Hwang, D. K.; Lim, S. W.; Myoung, J. M. Effects of Growth Temperature on the Properties of ZnO/GaAs Prepared by Metalorganic Chemical Vapor Deposition. J. Cryst. Growth 2003, 250, 437-443.

26. Chen, Y. J.; Shih, Y. Y.; Ho, C. H.; Du, J. H.; Fu, Y. P. Effect of Temperature on Lateral Growth of ZnO Grains Grown by MOCVD. Ceram. Int. 2010, 36, 69-73.

27. Malandrino, G.; Blandino, M.; Fragala, M. E.; Losurdo, M.; Bruno, G. Relationship between Nanostructure and Optical Properties of ZnO Thin Films. J. Phys. Chem. C 2008, 112, 9595-9599.

28. Park, J. Y.; Lee, D. J.; Yun, Y. S.; Moon, J. H.; Lee, B. T.; Kim, S. S. Temperature-Induced Morphological Changes of ZnO Grown by Metalorganic Chemical Vapor Deposition. J. Cryst. Growth 2005, 276, 158-164.

29. Consonni, V.; Feuillet, G.; Gergaud, P. Plasticity Induced Texture Development in Thick Polycrystalline Cdte: Experiments and Modeling. J. Appl. Phys. 2008, 103, 8. 
30. Jungk, T.; Hoffmann, A.; Soergel, E. Quantitative Analysis of Ferroelectric Domain Imaging with Piezoresponse Force Microscopy. Appl. Phys. Lett. 2006, 89, 3.

31. Kim, S.; Seol, D.; Lu, X. L.; Alexe, M.; Kim, Y. Electrostatic-Free Piezoresponse Force Microscopy. Sci. Rep. 2017, 7, 8.

32. Seomoon, K.; Lee, J.; Jang, P.; Jung, C.; Kim, K. H. Synthesis and Characterization of ZnO Thin Films Deposited Via Pe-Mocvd. Curr. Appl. Phys. 2011, 11, S26-S29.

33. Cusco, R.; Alarcon-Llado, E.; Ibanez, J.; Artus, L.; Jimenez, J.; Wang, B. G.; Callahan, M. J. Temperature Dependence of Raman Scattering in ZnO. Phys. Rev. B 2007, 75, 11.

34. Cong, G. W.; Wei, H. Y.; Zhang, P. F.; Peng, W. Q.; Wu, J. J.; Liu, X. L.; Jiao, C. M.; Hu, W. G.; Zhu, Q. S.; Wang, Z. G. One-Step Growth of ZnO from Film to Vertically Well-Aligned Nanorods and the Morphology-Dependent Raman Scattering. Appl. Phys. Lett. 2005, 87, 3.

35. Thompson, C. V. Structure Evolution During Processing of Polycrystalline Films. Annu. Rev. Mater. Sci. 2000, 30, 159-190.

36. Glas, F. Critical Dimensions for the Plastic Relaxation of Strained Axial Heterostructures in FreeStanding Nanowires. Phys. Rev. B 2006, 74, 4.

37. Zak, A. K.; Majid, W. H. A.; Abrishami, M. E.; Yousefi, R. X-Ray Analysis of ZnO Nanoparticles by Williamson-Hall and Size-Strain Plot Methods. Solid State Sci. 2011, 13, 251-256.

38. Mote, V. D.; Purushotham, Y.; Dole, B. N. Williamson-Hall Analysis in Estimation of Lattice Strain in Nanometer-Sized Zno Particles. J. Theor. Appl. Phys. 2012, 6, 6.

39. Ferrari, A. C.; Robertson, J., Interpretation of Raman Spectra of Disordered and Amorphous Carbon. Phys. Rev. B 2000, 61, 14095-14107.

40. Guillemin, S.; Appert, E.; Roussel, H.; Doisneau, B.; Parize, R.; Boudou, T.; Bremond, G.; Consonni, V., Controlling the Structural Properties of Single Step, Dip Coated Zno Seed Layers for Growing Perfectly Aligned Nanowire Arrays. J. Phys. Chem. C 2015, 119, 21694-21703.

41. Munuera, C.; Zuniga-Perez, J.; Rommeluere, J. F.; Sallet, V.; Triboulet, R.; Soria, F.; MunozSanjose, V.; Ocal, C. Morphology of ZnO Grown by Mocvd on Sapphire Substrates. J. Cryst. Growth 2004, 264, 70-78.

42. Meyer, B. K., Alves, H.; Hofmann, D. M.; Kriegseis, W.; Forster, D.; Bertram, F.; Christen, J.; Hoffmann, A.; Strassburg, M.; Dworzak, M.; Haboeck, U.; Rodina, A.V. Bound Exciton and DonorAcceptor Pair Recombinations in ZnO. Phys. Status Solidi B-Basic Solid State Phys. 2004, 241, 231-260. 43. Wagner, M. R., Callsen, G.; Reparaz, J. S.; Schulze, J. H.; Kirste, R.; Cobet, M.; Ostapenko, I. A.; Rodt, S.; Nenstiel, C.; Kaiser, M.; Hofmann, A.; Rodina, A. V.; Phillips, M. R.; Lautenschlager, S.; Eisermann, S.; Meyer, B. K. Bound Excitons in ZnO: Structural Defect Complexes Versus Shallow Impurity Centers. Phys. Rev. B 2011, 84, 18.

44. Mohammadbeigi, F.; Kumar, E. S.; Alagha, S.; Anderson, I.; Watkins, S. P. Carbon Related Donor Bound Exciton Transitions in ZnO Nanowires. J. Appl. Phys. 2014, 116, 7.

45. Lavrov, E. V.; Herklotz, F.; Weber, J. Identification of Two Hydrogen Donors in ZnO. Phys. Rev. B 2009, 79, 13.

46. Heinhold, R.; Neiman, A.; Kennedy, J. V.; Markwitz, A.; Reeves, R. J.; Allen, M. W. HydrogenRelated Excitons and Their Excited-State Transitions in ZnO. Phys. Rev. B 2017, 95, 13.

47. Cossuet, T.; Donatini, F.; Lord, A. M.; Appert, E.; Pernot, J.; Consonni, V. Polarity-Dependent High Electrical Conductivity of ZnO Nanorods and Its Relation to Hydrogen. J. Phys. Chem. C 2018, 122, 22767-22775.

48. Sieber, B.; Addad, A.; Szunerits, S.; Boukherroub, R. Stacking Faults-Induced Quenching of the Uv Luminescence in ZnO. J. Phys. Chem. Lett. 2010, 1, 3033-3038.

49. Yang, S.; Kuo, C. C.; Liu, W. R.; Lin, B. H.; Hsu, H. C.; Hsu, C. H.; Hsieh, W. F. Photoluminescence Associated with Basal Stacking Faults in C-Plane ZnO Epitaxial Film Grown by Atomic Layer Deposition. Appl. Phys. Lett. 2012, 100, 4.

50. Khranovskyy, V.; Eriksson, M. O.; Radnoczi, G. Z.; Khalid, A.; Zhang, H.; Holtz, P. O.; Hultman, L.; Yakimova, R. Photoluminescence Study of Basal Plane Stacking Faults in ZnO Nanowires. Physica $B$ 2014, 439, 50-53. 
51. Lyons, J. L.; Varley, J. B.; Steiauf, D.; Janotti, A.; Van de Walle, C. G. First-Principles Characterization of Native-Defect-Related Optical Transitions in ZnO. J. Appl. Phys. 2017, 122, 12.

52. Frodason, Y. K.; Johansen, K. M.; Bjorheim, T. S.; Svensson, B. G.; Alkauskas, A. Zn VacancyDonor Impurity Complexes in ZnO. Phys. Rev. B 2018, 97, 8.

53. Wander, A.; Harrison, N. M. An Ab Initio Study of ZnO(10-10). Surf. Sci. 2000, 457, L342-L346.

54. Wander, A.; Schedin, F.; Steadman, P.; Norris, A.; McGrath, R.; Turner, T. S.; Thornton, G.; Harrison, N. M. Stability of Polar Oxide Surfaces. Phys. Rev. Lett. 2001, 86, 3811-3814.

55. Kim, D. S.; Gosele, U.; Zacharias, M. Surface-Diffusion Induced Growth of ZnO Nanowires. J. Cryst. Growth 2009, 311, 3216-3219.

56. Hinchet, R.; Lee, S.; Ardila, G.; Montes, L.; Mouis, M.; Wang, Z. L. Performance Optimization of Vertical Nanowire- Based Piezoelectric Nanogenerators. Adv. Funct. Mater. 2014, 24, 971-977.

57. Michallon, J.; Bucci, D.; Morand, A.; Zanuccoli, M.; Consonni, V.; Kaminski-Cachopo, A. Light Trapping in ZnO Nanowire Arrays Covered with an Absorbing Shell for Solar Cells. Opt. Express 2014, 22, A1174-A1189.

58. Michallon, J.; Bucci, D.; Morand, A.; Zanuccoli, M.; Consonni, V.; Kaminski-Cachopo, A. Light Absorption Processes and Optimization of $\mathrm{ZnO} / \mathrm{CdTe}$ Core-Shell Nanowire Arrays for Nanostructured Solar Cells. Nanotechnology 2015, 26, 13.

59. Zuniga-Perez, J., Consonni, V.; Lymperakis, L.; Kong, X.; Trampert, A.; Fernandez-Garrido, S.; Brandt, O.; Renevier, H.; Keller, S.; Hestroffer, K.; Wagner, M. R.; Reparaz, J. S.; Akyol, F.; Rajan, S.; Rennesson, S.; Palacios, T.; Feuillet, G. Polarity in Gan and ZnO: Theory, Measurement, Growth, and Devices. Appl. Phys. Rev. 2016, 3, 100.

60. de la Mata, M.; Zamani, R. R.; Marti-Sanchez, S.; Eickhoff, M.; Xiong, Q. H.; Morral, A. F. I.; Caroff, P.; Arbiol, J. The Role of Polarity in Nonplanar Semiconductor Nanostructures. Nano Lett. 2019, 19, 3396-3408.

61. Consonni, V.; Sarigiannidou, E.; Appert, E.; Bocheux, A.; Guillemin, S.; Donatini, F.; Robin, I. C.; Kioseoglou, J.; Robaut, F. Selective Area Growth of Well-Ordered ZnO Nanowire Arrays with Controllable Polarity. ACS Nano 2014, 8, 4761-4770.

62. Lautenschlaeger, S.; Sann, J.; Volbers, N.; Meyer, B. K.; Hoffmann, A.; Haboeck, U.; Wagner, M. R. Asymmetry in the Excitonic Recombinations and Impurity Incorporation of the Two Polar Faces of Homoepitaxially Grown ZnO Films. Phys. Rev. B 2008, 77, 5.

63. Tao, R.; Mouis, M.; Ardila, G. Unveiling the Influence of Surface Fermi Level Pinning on the Piezoelectric Response of Semiconducting Nanowires. Adv. Electron. Mater. 2018, 4, 9. 
For Table of Contents Only

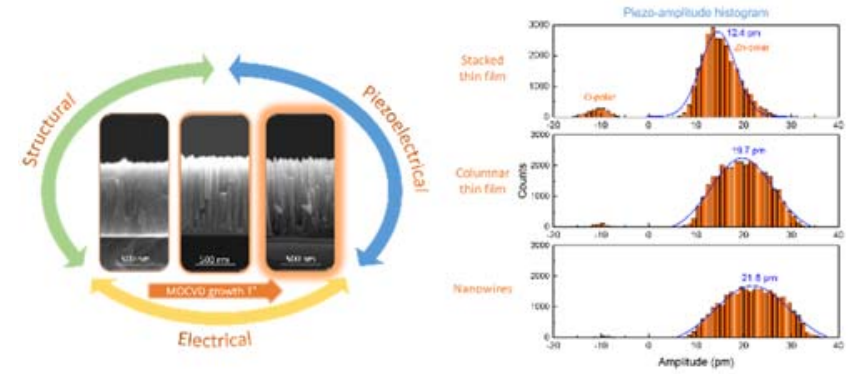

